\title{
Expermental Investigation of Turbulent Boundary Layer Flow Over Finned Surface
}

m.a Halawa, M. A El-Samanoudy

\begin{abstract}
This paper is concerned with the measurement of the velocity distribution and determination of the local skin friction coefficient for turbulent boundary layer developing on smooth and finned surfaces. The finned surface has a uniform roughness, which takes the shape of sinewave fins arranged in the transvers direction of ttie flow. The results indicated that the value of sldn friction coefficient is greatly increased for the finned surface case. The Reynolds number has no effect on the turbulent velocity profiles through the overlap region, when it was represented using the inner-law variables $u^{* *} \square$ and $\mathrm{y}^{\prime *}$ " for both the finned and the smooth surfaces
\end{abstract}

